\title{
Heifer Production on Rangeland and Seeded Forages in the Northern Great Plains
}

\author{
M. R. Haferkamp, ${ }^{1}$ M. D. MacNeil, ${ }^{2}$ \\ E. E. Grings, ${ }^{3}$ and K. D. Klement ${ }^{1}$ \\ Authors are ${ }^{1}$ Rangeland Scientists, ${ }^{2}$ Research Geneticist, and ${ }^{3}$ Research Animal Scientist, \\ USDA-ARS, Fort Keogh Livestock and Range Research Laboratory, Miles City, MT 59301.
}

\begin{abstract}
Integrating use of seeded perennial cool-season grass pastures with native rangeland can increase available forage and provide a high plane of nutrition for grazing livestock. Our objective was to compare performance of yearling beef heifers grazing native rangeland with those grazing an integrated system that included seeded forages. Twice-replicated, 3-ha pastures seeded to either 'Rosana' western wheatgrass (Pascopyron smithii [Rydb.] A. Love), 'Luna' pubescent wheatgrass (Elytrigia intermedia [Host] Nevski), or 'Hycrest' crested wheatgrass (Agropyron cristatum [L.] Gaertn. ssp. desertorum [Fisch. ex Link] A. Love) were grazed in spring, whereas twice-replicated, 3.24-ha pastures seeded to either 'Alkar' tall wheatgrass (Thinopyrum ponticum [Podp.] ZW Liu \& RC Wang), 'NewHy' hybrid wheatgrass (Elymus hoffmannii KB Jensen \& KH Asay), 'Bozoisky' Russian wildrye (Psathyrostachys juncea [Fisch.] Nevski.), or 'Prairieland' Altai wildrye (Leymus angustus [Trin.] Pilger) were grazed in autumn. Native rangeland was grazed during summer in the integrated system and spring, summer, and autumn in the rangeland treatment. Heifers exhibited greater weight gains on seeded pastures than on native rangeland in spring and autumn of most years. In 2 out of 3 years, heifers that grazed native rangeland during spring gained more $\left(P=0.012\right.$ for gain head ${ }^{-1}$ and $P=0.021$ for gain head $\left.{ }^{-1} \mathrm{day}^{-1}\right)$ while grazing native rangeland during summer than heifers that grazed seeded pastures in spring. Spring + summer gains averaged (mean \pm SE) $0.56 \pm 0.01 \mathrm{~kg} \cdot \mathrm{head}^{-1} \cdot \mathrm{d}^{-1}$ and $73.1 \pm 1.6 \mathrm{~kg} \cdot \mathrm{head}{ }^{-1}$. Livestock managers need to consider their livestock marketing and management strategies when using seeded pastures for seasonal grazing.
\end{abstract}

\section{Resumen}

La integración del uso de praderas sembradas de zacates perennes de estación fría con pastizal nativo puede incrementar el forraje disponible y proveer un buen plano nutricional para el ganado en apacentamiento. Nuestro objetivo fue comparar el comportamiento productivo de vaquillas de ganado de carne de año apacentando un pastizal nativo con vaquillas apacentando un sistema integrado que incluía forrajes sembrados. Praderas de 3 ha, repetidas dos veces, sembradas con "Western wheatgrass" (Pascopyron smithii [Rydb.] A. Love) variedad 'Rosana' y "Pubescent wheatgrass" (Elytrigia intermedia [Host] Nevski) variedad 'Luna' o con "Crested wheatgrass” (Agropyron cristatum [L.] Gaertn. ssp. desertorum [Fisch. ex Link] A. Love) variedad 'Hycrest' fueron apacentadas en primavera, mientras que praderas de 3.24 ha, repetidas dos veces, sembradas con "Tall wheatgrass" (Thinopyrum ponticum [Podp.] ZW Liu \& RC Wang) variedad 'Alkar', "Hybrid wheatgrass" (Elymus hoffmannii KB Jensen \& KH Asay) variedad 'NewHy', "Russian wildrye” (Psathyrostachys juncea [Fisch.] Nevski.) variedad 'Bozoisky' o con "Altai wildrye" (Leymus angustus [Trin.] Pilger) variedad 'Prairieland' fueron apacentadas en otoño; en el sistema integrado, el pastizal nativo fue apacentado en verano y en el tratamiento de solo pastizal se apacentó en primavera, verano y otoño. En la mayoría de los años, las vaquillas mostraron mayores ganancias de peso en las praderas sembradas que los obtenidos en el pastizal nativo en primavera y otoño. En 2 de 3 años, la vaquillas que apacentaron el pastizal nativo durante la primavera, ganaron más peso $\left(P=0.012\right.$ para ganancia cabeza ${ }^{-1}$ y $P=0.021$ para la ganancia cabeza ${ }^{-1}$ dia $\left.^{-1}\right)$ cuando apacentaban el $^{2}$ pastizal en verano que las vaquillas que apacentaron en primavera las praderas sembradas. Las ganancias de primavera + verano promediaron $0.56 \pm 0.01 \mathrm{~kg} \cdot \mathrm{cabeza}^{-1} \cdot \mathrm{d}^{-1}$ y $73.1 \pm 1.6 \mathrm{~kg} \cdot \mathrm{cabeza}^{-1}$. Cuando utilicen praderas sembradas para el apacentamiento estacional, los manejadores de ganado necesitan considerar su mercadeo del ganado y estrategias de manejo.

Key Words: 'Alkar', 'Bozoisky', 'Hycrest', 'Luna', 'NewHy', 'Prairieland', 'Rosana', native rangeland, crude protein, digestibility, standing crop

\section{INTRODUCTION}

This article is a contribution from the USDA-ARS and Montana Agr. Exp. Sta., Miles City, MT. The USDA-ARS, Northern Plains Area, is an equal opportunity/affirmative action employer, and all agency services are available without discrimination.

Mention of any trade name or proprietary product does not constitute a guarantee or warranty by the authors or USDA-ARS, nor does it imply the approval of these products to the exclusion of others.

Correspondence: Marshall R. Haferkamp, 243 Fort Keogh Road, Miles City, MT 59301. Email: marshall@larrl.ars.usda.gov

Manuscript received 7 January 2005; manuscript accepted 15 June 2005.
Pastures seeded to perennial cool-season grasses may be used to reduce grazing pressure on native rangelands and provide quality forage for livestock at selected seasons (Wight et al. 1983). Seasonally, weight gains per head and per hectare are often, but not always, greater on seeded pastures compared with native rangeland (Smoliak 1968; Smoliak and Slen 1974; Kilcher and Lawrence 1979; Karn et al. 1999). Observed increases in animal performance often result from increases in 


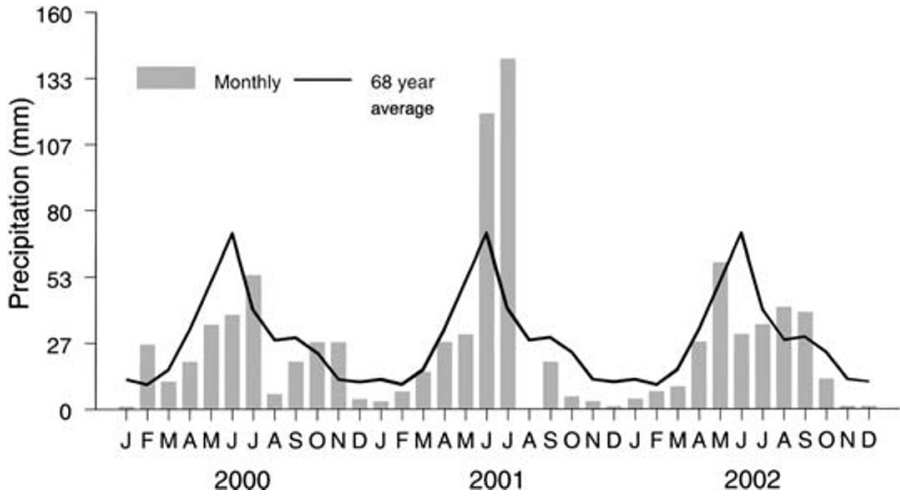

Figure 1. Precipitation recorded at the Frank Wiley Airfield located about $4 \mathrm{~km}$ from the study site (bars); long-term average from Frank Wiley Airfield (NOAA 2000-2002).

both quantity and quality of forage available. However, often increased gains per hectare also result from higher stocking rates used on seeded pastures compared with native rangeland (Hart et al. 1983; Adams et al. 1989; Hofmann et al. 1993). Variable animal responses have been observed over both time (e.g., years) and space (e.g., sites) so research needs to be conducted within a variety of climate and vegetation types (Willms and Jefferson 1993). Our objective was to compare performance of yearling heifers grazing native rangeland with contemporary animals grazing seeded forages in an integrated system. Specific hypotheses tested effects on quantity and quality of standing crop, diet quality, and weight change of heifers attributable to differences among forage resources, temporal variation, and associated interactions.

\section{MATERIALS AND METHODS}

\section{Study Site}

Research was conducted at Fort Keogh Livestock and Range Research Laboratory (lat $46^{\circ} 22^{\prime} \mathrm{N}$, long $105^{\circ} 5^{\prime} \mathrm{W}$ ) near Miles City, Montana. Elevation on the study site is about $720 \mathrm{~m}$. Regional topography varies from rolling hills to broken badlands with small, intersecting ephemeral streams flowing into rivers in broad, nearly level valleys. This area is representative of the semiarid, mixed-grass prairie of the Northern Great Plains. Indigenous vegetation on the 22 500-ha research station is a grama-needlegrass-wheatgrass (Bouteloua-Stipa-Agropyron) mix (Küchler 1964). Annual precipitation averages $343 \mathrm{~mm}$, with about $75 \%$ typically received from April through September (Fig. 1). Daily temperatures range from $>38^{\circ} \mathrm{C}$ during summer to $<-40^{\circ} \mathrm{C}$ during winter. The average frost-free growing season is 150 days. Soils on the spring-seeded pasture site are a composite of Glendive loam (coarse-loamy, mixed, superactive, calcareous, frigid Aridic Ustifluvents) and Havre loam (fine-loamy, mixed, superactive, calcareous, frigid Aridic Ustifluvents) with $0 \%-2 \%$ slopes. Soils on the autumn-seeded pasture site are a composite of Marias clay and silty clay (fine montmorillonitic, frigid Chromic Udic Haplusterts) with $0 \%-$ $2 \%$ slopes. The Marias soils are moderately to strongly alkaline with $\mathrm{pH}$ ranging from 7.4 to 9.0 .

\section{Treatments}

Seeded forages were planted in randomized-block designs with 2 replications. Three seeded forages ('Rosana' western wheatgrass, Pascopyron smithii [Rydb.] A. Love; 'Luna' pubescent wheatgrass, Elytrigia intermedia [Host] Nevski; and 'Hycrest' crested wheatgrass Agropyron cristatum [L.] Gaertn. ssp. desertorum [Fisch. ex Link] A. Love) and native rangeland were grazed in spring. Seeded pastures grazed in spring were 3.0-ha. Four seeded forages ('Prairieland' Altai wildrye, Leymus angustus [Trin.] Pilger; 'Alkar' tall wheatgrass, Thinopyrum ponticum [Podp.] ZW Liu \& RC Wang; 'Bozoisky' Russian wildrye, Psathyrostachys juncea [Fisch.] Nevski; and 'NewHy' hybrid wheatgrass Elymus hoffmannii KB Jensen \& KH Asay) and native rangeland were grazed in autumn. Seeded pastures grazed in autumn were 3.24-ha. Two pastures were planted to each forage cultivar in autumn and rested for a minimum of 1 year to ensure successful stand establishment. Stand establishment was deemed successful in all pastures except 1 of the 2 Prairieland pastures, which was subsequently eliminated from the study. Use of a single rangeland pasture in spring and autumn and failure of 1 replication of Prairieland in autumn resulted in each experiment being an incomplete block design. The 3 sampled sites in the rangeland pasture each included an upland, sideslope, and bottomland and were treated as replications of the native rangeland treatment in spring and autumn.

Native rangeland was also grazed in summer with all native rangeland pastures located about $17.5 \mathrm{~km}$ from the seeded pastures. Summer grazed native rangeland consisted of 6 adjoining pastures ranging in size from 266 to 350 ha.

All replications of seeded pastures were grazed continuously with 7 heifers during their respective grazing period. Spring grazing periods ranged from about 30 to 45 days beginning in mid to late April. Spring grazing trials were terminated by 1 June so heifers could be exposed for breeding to assigned sires in accordance with breeding protocols for genetic studies. Autumn pastures were grazed for 30-55 days beginning in early September. Length of autumn grazing periods varied among years and forage species depending on available forage (see Results). Heifers on native rangeland during spring and autumn grazed with contemporary heifers. Similarly, during summer, heifers grazed with cow-calf pairs in the breeding herds. Livestock were handled according to protocol approved by the Fort Keogh Livestock and Range Research Laboratory Animal Care Committee.

\section{Field Measurements}

Cattle used in the study were registered Line 1 Hereford (Bos taurus) yearling heifers that were approximately 13 months of age at the beginning of the spring grazing period. At the beginning of each grazing season, heifers were weighed after an overnight shrink, stratified by initial weight into groups of 7 heifers, and within strata randomly assigned to the 6 spring pastures and to the single native rangeland pasture. Initial weights of animals averaged $312 \mathrm{~kg}(\mathrm{SD}=34)$ in $2000,325 \mathrm{~kg}$ $(\mathrm{SD}=32)$ in 2001 , and $302 \mathrm{~kg}(\mathrm{SD}=28)$ in 2002. Thereafter, weights were obtained at the end of the spring grazing period and at the start and end of both the summer and autumn grazing periods. In autumn, heifers were again restratified by weight and randomly reassigned within strata to the autumn 
pastures. Heifers were treated with Ivermectin (Merck \& Co Inc, Whitehouse Station, NJ) before the study for parasite control and received an ear tag with fly repellent at the beginning of the grazing season. Trace mineralized salt was available at all times in all pastures. Weight gains are presented on both a per head and per hectare basis.

\section{Standing Crop}

Standing crops were estimated in seeded pastures during weeks that livestock were weighed, by clipping forage to ground level in 15 rectangular $0.25-\mathrm{m}^{2}$ quadrats. The 15 quadrats were spaced equally (by pacing) along a diagonal line across each pasture, and a different diagonal was used for the pre- and postgrazing clipping. Clipped biomass was separated into seeded species (live + dead) and nonseeded species (live + dead). Standing crop of native rangeland pastures was estimated in a single 550-ha pasture in spring and autumn and 2 pastures (550 and 314 ha) in summer because resources were not available to sample all 6 native rangeland pastures used during summer. Seasonal estimates used for these pastures were derived for upland, sideslope, and bottomland sites by clipping 15 rectangular $0.10-\mathrm{m}^{2}$ quadrats on each of 9 sites (i.e., 3 each, upland, sideslope, and bottomland sites). Sites were selected as representative of the large pasture, and quadrats were located randomly within each sample site. Native vegetation was clipped to ground level and sorted into grasses and forbs. All harvested biomass was dried to a constant weight in a forced draft oven at $60^{\circ} \mathrm{C}$ and weighed.

\section{Diets}

Esophageal extrusa was sampled at least once during each spring, summer, and autumn grazing period (i.e., seeded and native rangeland), near the middle of each seasonal grazing period to estimate diet quality. Each pasture was sampled with 3 mature (i.e., 3-5 years of age), esophageally cannulated, crossbred cows. Cows had been cannulated at 2 months of age and were experienced with the extrusa sampling process. Cows were penned the day before sampling at about 1600 hours with water but no feed available. Collections were made the following morning beginning at 0700 hours using vinyl mesh-bottomed collection bags. Collection periods lasted from 20 to 30 minutes and were followed immediately by a collection in a second pasture. Esophageal extrusa samples were thoroughly mixed by hand and freeze-dried for laboratory analyses. Between sampling periods, cows were kept in larger pastures with a variety of range sites but not with the grass cultivars being assessed. However, cows had been previously exposed to most of the plant species present in the experimental pastures.

\section{Laboratory Analysis}

All esophageal extrusa samples and standing crop samples were ground to pass through a 1-mm screen in a Wiley mill (Arthur H. Thomas Co, Philadelphia, PA). Total nitrogen (N) was determined on a C-N analyzer (Carlo-Erba, CE Elantech, Inc, Lakewood, NJ) after roller-grinding (Mortenson 2003). Data are presented as percentage crude protein $(\mathrm{CP})$ in standing
Table 1. Stocking pressures and beginning and ending standing crops (means $\pm \mathrm{SE}$ ) for seeded and native rangeland pastures at Miles City, Montana.

\begin{tabular}{|c|c|c|c|c|c|}
\hline \multirow[b]{2}{*}{ Season } & \multirow[b]{2}{*}{ Year } & \multirow[b]{2}{*}{ Pasture } & \multirow[b]{2}{*}{$\mathrm{AUD}^{1} \cdot \mathrm{ha}^{-1}$} & \multicolumn{2}{|c|}{ Standing crop $\left(\mathrm{kg} \cdot \mathrm{ha}^{-1}\right)$} \\
\hline & & & & $\ln$ & Out \\
\hline \multirow[t]{12}{*}{ Spring } & 2000 & Hycrest & 100.3 & $1411 \pm 275$ & $754 \pm 275$ \\
\hline & & Luna & 100.3 & $1425 \pm 275$ & $333 \pm 324$ \\
\hline & & Rosana & 100.3 & $1536 \pm 275$ & $743 \pm 275$ \\
\hline & & Native & 3.1 & $917 \pm 224$ & $958 \pm 224$ \\
\hline & 2001 & Hycrest & 65.3 & $1255 \pm 275$ & $1444 \pm 275$ \\
\hline & & Luna & 65.3 & $1966 \pm 275$ & $1309 \pm 275$ \\
\hline & & Rosana & 65.3 & $1235 \pm 275$ & $985 \pm 275$ \\
\hline & & Native & 1.1 & $660 \pm 224$ & $1029 \pm 224$ \\
\hline & 2002 & Hycrest & 86.3 & $2592 \pm 275$ & $1412 \pm 275$ \\
\hline & & Luna & 86.3 & $1840 \pm 275$ & $1552 \pm 275$ \\
\hline & & Rosana & 86.3 & $1716 \pm 275$ & $1116 \pm 275$ \\
\hline & & Native & 3.1 & $717 \pm 224$ & $764 \pm 224$ \\
\hline \multirow[t]{15}{*}{ Autumn } & 2000 & Alkar & 77.8 & $3217 \pm 286$ & $2079 \pm 286$ \\
\hline & & Bozoisky & 62.6 & $1164 \pm 286$ & $512 \pm 286$ \\
\hline & & NewHy & 77.8 & $1981 \pm 286$ & $870 \pm 286$ \\
\hline & & Prairieland & 62.6 & $2013 \pm 404$ & $1187 \pm 404$ \\
\hline & & Native & 30.8 & $876 \pm 234$ & $646 \pm 234$ \\
\hline & 2001 & Alkar & 121.0 & $5086 \pm 286$ & $2687 \pm 286$ \\
\hline & & Bozoisky & 108.0 & $1616 \pm 286$ & $451 \pm 286$ \\
\hline & & NewHy & 121.0 & $2987 \pm 286$ & $1050 \pm 286$ \\
\hline & & Prairieland & 121.0 & $4071 \pm 404$ & $2218 \pm 404$ \\
\hline & & Native & 10.9 & $1028 \pm 234$ & $682 \pm 234$ \\
\hline & 2002 & Alkar & 125.3 & $4154 \pm 286$ & $2151 \pm 286$ \\
\hline & & Bozoisky & 79.9 & $1133 \pm 286$ & $360 \pm 286$ \\
\hline & & NewHy & 125.3 & $1975 \pm 286$ & $647 \pm 286$ \\
\hline & & Prairieland & 125.3 & $2832 \pm 404$ & $1376 \pm 404$ \\
\hline & & Native & 4.2 & $789 \pm 234$ & $556 \pm 234$ \\
\hline
\end{tabular}

${ }^{1}$ Animal unit days.

crops (dry matter basis) and diets (organic matter basis). In vitro digestible organic matter (IVDOM) was determined on esophageal extrusa samples using a modified Tilly and Terry (1963) technique (White et al. 1981). Data are presented as percentage of IVDOM in diets.

\section{Data Summarization and Analysis}

Weight gains of the heifers were expressed per head, per head per day, and per hectare. Gain per hectare was calculated as stocking pressure (animal unit days, $\left[\right.$ AUD] $\cdot \mathrm{ha}^{-1}$ ) (Table 1) multiplied by the daily gain $\cdot$ head $^{-1}$. In all seasons, the calculated stocking rate on native rangeland were based on all $\mathrm{AU}$ in those pastures.

Analyses of variance were conducted using PROC MIXED of SAS (2002) to test fixed effects of treatments, years, dates, and interactions among them. Variation among pastures within treatments (i.e., replications) was considered as a random effect. Differences among treatments were tested with variation among pastures within treatments, and other effects were tested with the residual variance. Data from the spring and autumn seasons were analyzed separately. Dependent variables were 
Table 2. Least-square means \pm standard error for standing crop on seeded pastures and native rangeland at Miles City, Montana.

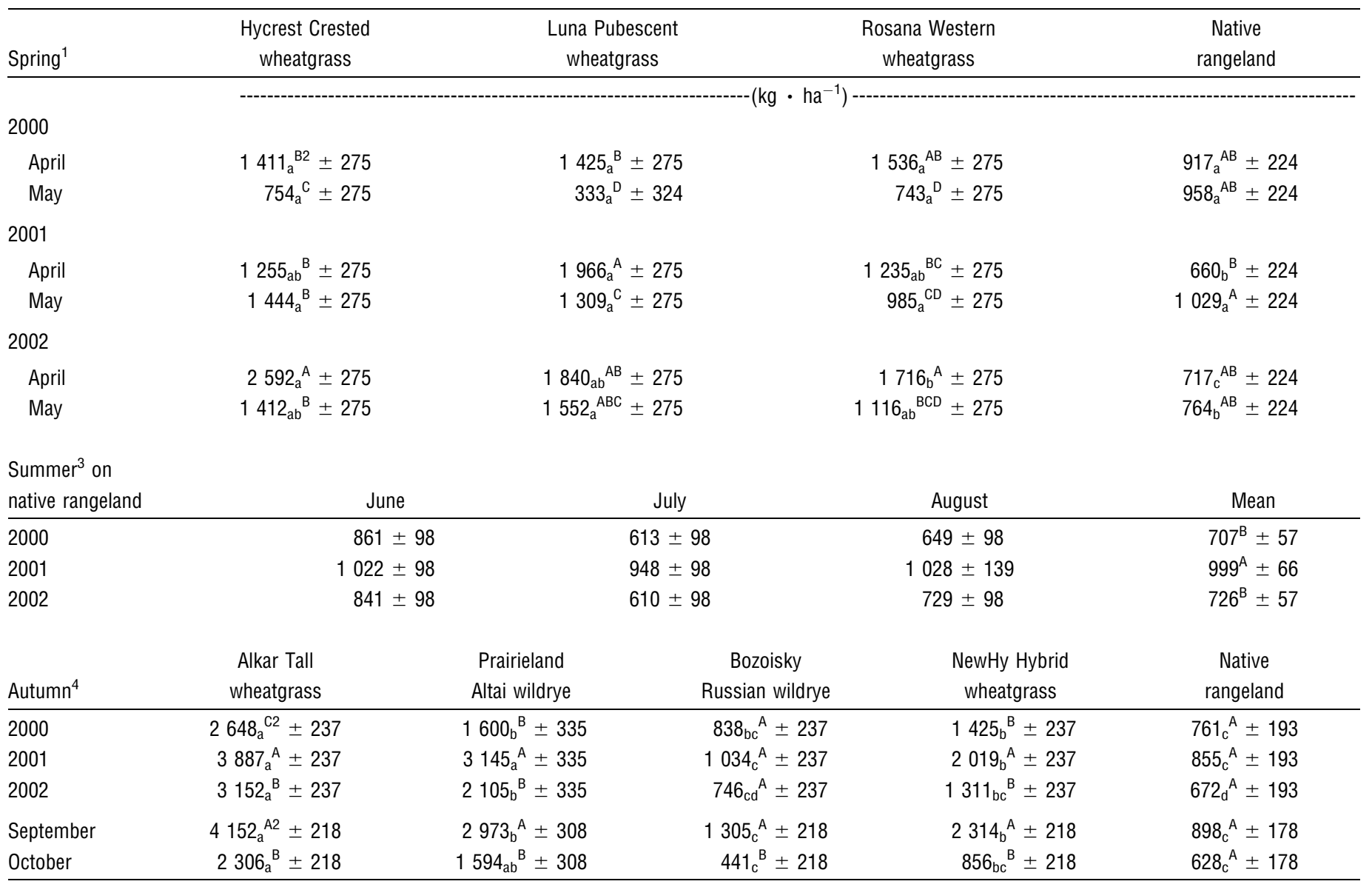

${ }^{1}$ Significant pasture-by-year-by-date interaction $(P=0.012)$ for spring standing crop.

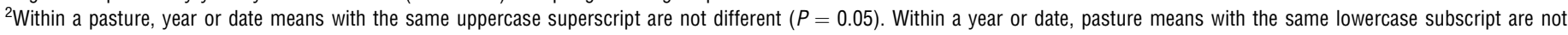
different $(P=0.05)$.

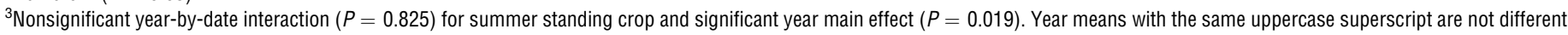
$(P=0.05)$.

${ }^{4}$ Significant pasture-by-year interaction $(P=0.001)$ for autumn standing crop and significant pasture-by-date interaction $(P=0.001)$ for autumn standing crop.

measures of standing crop, forage quality, diet quality, and animal performance.

Quantity and quality of forage on native rangeland grazed during summer were analyzed with a model that included fixed effects of years and dates and their interaction and the random effect of pasture nested within year and date. Weight gains observed during summer were evaluated for carryover effects of the treatments applied during spring with the model used to analyze weight gain observed during spring. The least significant difference (LSD) method protected by a prior F-test $(P \leq 0.05)$ was used for comparing means.

\section{RESULTS}

\section{Conditions During Study}

Precipitation during April, May, and June is important for determining forage production in the Northern Great Plains. Precipitation was below average during this period for much of this study (Fig. 1). The largest precipitation events occurred during June and July 2001.
Stocking pressure was consistently greater on seeded pastures in spring, ranging from 65 to 100 AUD $\cdot \mathrm{ha}^{-1}$ compared with 1.1 to $3.1 \mathrm{AUD} \cdot \mathrm{ha}^{-1}$ on native rangeland (Table 1 ). Standing crops during spring were consistently less after grazing (May) than before (April) on seeded pastures in 2000 $(P=0.004)$ but less only for Luna in $2001(P=0.004)$ and for Hycrest $(P=0.001)$ and Rosana $(P=0.008)$ in 2002 (Table $2)$. Standing crops on spring native rangeland differed little from April through May in $2000(P=0.821)$ and 2002 $(P=0.798)$ but increased significantly in $2001(P=0.044)$ (Table 2). Standing crops were similar before and after grazing on summer rangelands $(P=0.131)$ (Table 2$)$. During the 90day summer grazing season, pastures were stocked at a moderately heavy stocking rate for the site based on National Resources Conservation Service (NRCS) recommendations for these range sites (Soil Conservation Service 1983). During autumn, stocking pressure ranged from 63 to $125 \mathrm{AUD} \cdot \mathrm{ha}^{-1}$ on seeded pastures and from 4.4 to 31 AUD $\cdot$ ha $^{-1}$ on native rangeland (Table 1). Standing crops were less after grazing on seeded pastures $(P=0.001)$ but not rangeland $(P=0.077)$ in autumn (Table 2). 
Table 3. Least-square means \pm standard error for crude protein contained in standing crop on seeded pastures and native rangeland at Miles City, Montana.

\begin{tabular}{|c|c|c|c|c|c|}
\hline Spring $^{1}$ & $\begin{array}{r}\text { Hycrest Cres } \\
\text { wheatgras }\end{array}$ & \multicolumn{2}{|c|}{$\begin{array}{c}\text { Luna Pubescent } \\
\text { wheatgrass }\end{array}$} & $\begin{array}{c}\text { Rosana Western } \\
\text { wheatgrass }\end{array}$ & $\begin{array}{c}\text { Native } \\
\text { rangeland }\end{array}$ \\
\hline & \multirow{2}{*}{\multicolumn{3}{|c|}{$14.2_{\mathrm{a}}^{\mathrm{A} 2} \pm 0.8$}} & 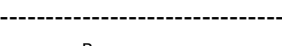 & 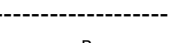 \\
\hline 2000 & & & & $10.9_{b}^{B} \pm 0.8$ & $8.6_{\mathrm{c}}{ }^{\mathrm{B}} \pm 0.6$ \\
\hline 2001 & \multicolumn{2}{|c|}{$15.0^{A}{ }^{A} \pm 0.8$} & $14.0_{\mathrm{a}}^{\mathrm{A}} \pm 0.8$ & $13.7_{\mathrm{a}}^{\mathrm{A}} \pm 0.8$ & $10.1_{b}{ }^{A} \pm 0.6$ \\
\hline 2002 & \multicolumn{2}{|c|}{$13.8_{\mathrm{a}}^{\mathrm{A}} \pm 0.8$} & $12.6_{a}{ }^{B} \pm 0.8$ & $12.1_{\mathrm{a}}^{\mathrm{B}} \pm 0.8$ & $9.8_{\mathrm{b}}{ }^{\mathrm{A}} \pm 0.6$ \\
\hline Spring $^{3}$ & \multicolumn{4}{|c|}{ April } & May \\
\hline 2000 & \multicolumn{4}{|c|}{$12.0_{a}^{\mathrm{B}} \pm 0.4$} & $11.9_{a}^{B} \pm 0.5$ \\
\hline 2001 & \multicolumn{4}{|c|}{$13.9^{\mathrm{A}} \pm 0.4$} & $12.5_{\mathrm{b}}{ }^{\mathrm{AB}} \pm 0.4$ \\
\hline 2002 & \multicolumn{4}{|c|}{$11.1_{b}{ }^{C} \pm 0.4$} & $13.1_{a}^{A} \pm 0.4$ \\
\hline \multicolumn{6}{|l|}{ Summer $^{4}$ on } \\
\hline native rangeland & \multicolumn{2}{|c|}{ June } & July & August & Mean \\
\hline 2000 & \multicolumn{2}{|c|}{$8.4 \pm 0.4$} & $6.2 \pm 0.4$ & $5.1 \pm 0.4$ & $6.6^{B} \pm 0.2$ \\
\hline 2001 & \multicolumn{2}{|c|}{$9.7 \pm 0.4$} & $8.2 \pm 0.4$ & $7.4 \pm 0.6$ & $8.4^{\mathrm{A}} \pm 0.3$ \\
\hline 2002 & \multicolumn{2}{|c|}{$10.3 \pm 0.4$} & $6.9 \pm 0.4$ & $6.7 \pm 0.4$ & $7.9^{A} \pm 0.2$ \\
\hline Mean & \multicolumn{2}{|c|}{$9.5_{\mathrm{a}} \pm 0.2$} & $7.1_{\mathrm{b}} \pm 0.2$ & $6.4_{b} \pm 0.3$ & \\
\hline Autumn $^{5}$ & $\begin{array}{c}\text { Alkar Tall } \\
\text { wheatgrass }\end{array}$ & $\begin{array}{c}\text { Prairieland } \\
\text { Altai wildrye }\end{array}$ & $\begin{array}{c}\text { Bozoisky } \\
\text { Russian wildrye }\end{array}$ & $\begin{array}{c}\text { NewHy Hybrid } \\
\text { wheatgrass }\end{array}$ & $\begin{array}{c}\text { Native } \\
\text { rangeland }\end{array}$ \\
\hline \multicolumn{6}{|l|}{2000} \\
\hline September & $4.8^{\mathrm{b}}{ }^{\mathrm{A} 2} \pm 0.4$ & $8.7_{\mathrm{a}}^{\mathrm{A}} \pm 0.6$ & $7.7_{\mathrm{a}}^{\mathrm{D}} \pm 0.4$ & $5.8_{b}{ }^{B C} \pm 0.4$ & $5.3_{b}^{B} \pm 0.3$ \\
\hline October & $4.2_{c}^{\mathrm{B}} \pm 0.4$ & $6.0_{b}^{B} \pm 0.6$ & $9.0_{\mathrm{a}}^{\mathrm{BC}} \pm 0.4$ & $5.0_{b c}{ }^{C} \pm 0.4$ & $5.3_{b}^{B} \pm 0.3$ \\
\hline \multicolumn{6}{|l|}{2001} \\
\hline September & $5.2_{c}{ }^{A} \pm 0.4$ & $9.2^{A} \pm 0.6$ & $9.5_{\mathrm{a}}^{\mathrm{B}} \pm 0.4$ & $7.5_{b}{ }^{A} \pm 0.4$ & $7.4_{b}{ }^{A} \pm 0.3$ \\
\hline October & $4.5_{\mathrm{c}}^{\mathrm{AB}} \pm 0.4$ & $5.7_{\mathrm{bc}}{ }^{\mathrm{BC}} \pm 0.6$ & $8.4^{C D} \pm 0.4$ & $5.6 b^{B C}{ }^{B C} \pm 0.4$ & $5.7_{\mathrm{b}}^{\mathrm{B}} \pm 0.3$ \\
\hline \multicolumn{6}{|l|}{2002} \\
\hline September & $5.1_{\mathrm{c}}^{\mathrm{A}} \pm 0.4$ & $5.3_{\mathrm{bc}}^{\mathrm{BC}} \pm 0.6$ & $7.1_{\mathrm{a}}^{\mathrm{D}} \pm 0.4$ & $6.0_{a b c}{ }^{B} \pm 0.4$ & $6.4_{a b}{ }^{A} \pm 0.3$ \\
\hline October & $5.2_{c d}{ }^{A} \pm 0.4$ & $4.7_{d}^{C} \pm 0.6$ & $11.5_{\mathrm{a}}^{\mathrm{A}} \pm 0.4$ & $6.2_{c}{ }^{B} \pm 0.4$ & $7.9_{b}{ }^{A} \pm 0.3$ \\
\hline
\end{tabular}

${ }^{1}$ Significant pasture-by-year-by-date interaction $(P=0.01)$ for spring crude protein contents.

${ }^{2}$ Within a pasture, year or date means with the same uppercase superscript are not different $(P=0.05)$. Within a year or date, pasture means with the same lowercase subscript are not different $(P=0.05)$.

${ }^{3}$ Significant date-by-year interaction $(P=0.014)$ for spring crude protein contents. Within a date, year means with the same uppercase superscript are not different $(P=0.05)$. Within a year, date means with the same lowercase subscript are not different $(P=0.05)$.

${ }^{4}$ Nonsignificant date-by-year interaction $(P=0.256)$ for summer crude protein contents. Significant date $(P=0.001)$ and year $(P=0.002)$ main effects $(P=0.05)$. Year means with the same lowercase subscript are not different $(P=0.05)$, and year means with the same uppercase superscript are not different $(P=0.05)$.

${ }^{5}$ Significant pasture-by-year-by-date interaction $(P=0.001)$ for autumn crude protein contents.

\section{Standing Crops}

Spring. Standing crops at the time grazing began in spring were greater $(P=0.020)$ on seeded pastures $(1381 \pm 117$ $\left.\mathrm{kg} \cdot \mathrm{ha}^{-1}\right)$ than on native rangeland $\left(841 \pm 165 \mathrm{~kg} \cdot \mathrm{ha}^{-1}\right)$. Standing crops were similar among seeded species in 2000 , but greatest for Luna in 2001 and Hycrest in 2002 (Table 2). Composition of seeded grasses in the total standing crop declined $(P=0.001)$ from $75 \%$ of the standing crop in 2000 to $56 \%$ in 2001 and 2002 with no significant $(P=0.208)$ difference between April and May (data not shown).

Summer. Standing crop on native rangeland was similar among dates $(P=0.825)$. The standing crop was consistently greater $(P=0.019)$ during 2001 than in 2000 or 2002 (Table 2).
Autumn. Alkar standing crops were greater $(P=0.001)$ than for Bozoisky, NewHy, or native rangeland (Table 2). Prairieland standing crops were less than for Alkar in 2000 $(P=0.012)$ and $2002(P=0.012)$, and similar to NewHy in $2000(P=0.671)$ and $2001(P=0.055)$. Standing crops were also greater in 2001 compared to $2000(P=0.010)$ and 2002 $(P=0.002)$ for all except Bozoisky $(P=0.391)$ and native rangeland $(P=0.613)$. Seeded grasses contributed more than $90 \%$ of the standing crop in Alkar, Bozoisky, and NewHy pastures in contrast to $80 \%$ for Prairieland $(P=0.013)$.

\section{Forage Quality}

Spring. Crude protein concentrations were consistently greater $(P=0.021)$ for seeded pastures compared with native 
Table 4. Least square means \pm standard error for average crude protein contained in diets of esophageally fistulated cattle grazing seeded pastures and native rangeland at Miles City, Montana.

\begin{tabular}{|c|c|c|c|c|}
\hline Spring $^{1}$ & $\begin{array}{c}\text { Hycrest Crested } \\
\text { wheatgrass }\end{array}$ & $\begin{array}{c}\text { Luna Pubescent } \\
\text { wheatgrass }\end{array}$ & $\begin{array}{c}\text { Rosana Western } \\
\text { wheatgrass }\end{array}$ & $\begin{array}{c}\text { Native } \\
\text { rangeland }\end{array}$ \\
\hline & & 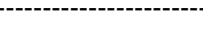 & & \\
\hline 2000 & $21.0_{\mathrm{a}}^{\mathrm{A} 2} \pm 2.2$ & $19.2_{a b}{ }^{A} \pm 2.4$ & $13.6_{b c}{ }^{B} \pm 2.1$ & $11.4_{c}^{A} \pm 3.0$ \\
\hline 2001 & $22.5^{\mathrm{A}} \pm 2.2$ & $20.5_{a b}{ }^{A} \pm 2.2$ & $21.5^{A} \pm 2.1$ & $13.4_{b}{ }^{A} \pm 3.0$ \\
\hline 2002 & $20.6_{a}^{A} \pm 2.1$ & $19.2_{a b}{ }^{A} \pm 2.1$ & $16.5_{a b}{ }^{A B} \pm 2.2$ & $12.1_{\mathrm{b}}{ }^{\mathrm{A}} \pm 3.0$ \\
\hline
\end{tabular}

Summer ${ }^{3}$ on

native rangeland

Jun

\begin{tabular}{|c|c|c|c|c|}
\hline 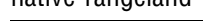 & & & o & \\
\hline 2000 & $8.4 \pm 0.2$ & $6.3 \pm 0.2$ & $6.5 \pm 0.3$ & $7.1^{C} \pm 0.1$ \\
\hline 2001 & $11.0 \pm 0.2$ & $8.5 \pm 0.2$ & $8.0 \pm 0.3$ & $9.2^{A} \pm 0.1$ \\
\hline 2002 & $10.2 \pm 0.2$ & $7.7 \pm 0.2$ & $6.8 \pm 0.2$ & $8.2^{B} \pm 0.1$ \\
\hline Mean & $9.9_{\mathrm{a}} \pm 0.1$ & $7.5_{b} \pm 0.1$ & $7.1_{b} \pm 0.2$ & \\
\hline
\end{tabular}

\begin{tabular}{|c|c|c|c|c|c|}
\hline Autumn ${ }^{4}$ & $\begin{array}{c}\text { Alkar Tall } \\
\text { wheatgrass }\end{array}$ & $\begin{array}{l}\text { Prairieland } \\
\text { Altai Wildrye }\end{array}$ & $\begin{array}{c}\text { Bozoisky Russian } \\
\text { wildrye }\end{array}$ & $\begin{array}{c}\text { NewHy Hybrid } \\
\text { wheatgrass }\end{array}$ & $\begin{array}{c}\text { Native } \\
\text { rangeland }\end{array}$ \\
\hline 2000 & $5.9_{b}{ }^{\mathrm{A} 2} \pm 1.1$ & $12.2^{\mathrm{A}} \pm 1.3$ & $8.4_{b}^{C} \pm 1.1$ & $6.5_{b}{ }^{B} \pm 1.1$ & $6.2_{b}{ }^{B} \pm 1.5$ \\
\hline 2001 & $6.1_{\mathrm{b}}^{\mathrm{A}} \pm 1.1$ & $11.3^{\mathrm{A}} \pm 1.3$ & $10.0^{\mathrm{B}} \pm 1.1$ & $8.3_{a b}{ }^{A} \pm 1.1$ & $8.6_{a b}{ }^{A} \pm 1.5$ \\
\hline 2002 & $7.2_{\mathrm{C}}^{\mathrm{A}} \pm 1.1$ & $11.5^{\mathrm{b}}{ }^{\mathrm{A}} \pm 1.4$ & $16.1^{\mathrm{A}} \pm 1.2$ & $9.7_{\mathrm{bc}}{ }^{\mathrm{A}} \pm 1.1$ & $8.4_{b c}{ }^{A} \pm 1.5$ \\
\hline
\end{tabular}

${ }^{1}$ Significant pasture-by-year interaction $(P=0.045)$ for spring pastures.

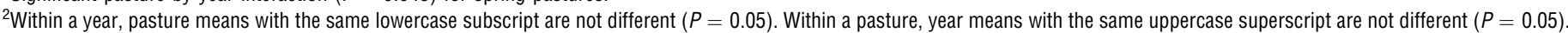

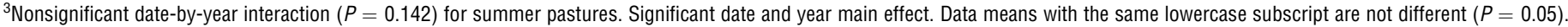

Year means with the same uppercase superscript are not different $(P=0.05)$.

${ }^{4}$ Significant pasture-by-year interaction $(P=0.001)$ for autumn pastures.

Table 5. Least-square means \pm standard error for average in vitro digestible organic matter contained in diets of esophageally fistulated cattle grazing seeded pastures and native rangeland at Miles City, Montana.

\begin{tabular}{lcccc}
\hline Spring & Hycrest Crested \\
wheatgrass & $\begin{array}{c}\text { Luna Pubescent } \\
\text { wheatgrass }\end{array}$ & $\begin{array}{c}\text { Rosana Western } \\
\text { wheatgrass }\end{array}$ & $\begin{array}{c}\text { Native } \\
\text { rangeland }\end{array}$ \\
\hline & $-1.7 \pm 1.6$ & $76.7 \pm 1.9$ & $67.7 \pm 1.4$ & $66.7 \pm 1.9$ \\
2001 & $77.4 \pm 1.5$ & $77.7 \pm 1.5$ & $76.2 \pm 1.4$ & $70.5 \pm 1.9$ \\
2002 & $71.9 \pm 1.4$ & $72.7 \pm 1.4$ & $70.4 \pm 1.5$ & $66.8 \pm 1.9$ \\
Mean & $74.7_{\mathrm{a}} \pm 1.0$ & $75.7_{\mathrm{a}} \pm 1.0$ & $71.4_{\mathrm{ab}} \pm 0.9$ & $68.0_{\mathrm{b}} \pm 1.3$
\end{tabular}

Summer ${ }^{2}$ on

native rangeland

\begin{tabular}{crr}
\multicolumn{1}{c}{ June } & \multicolumn{1}{c}{ July } & \multicolumn{1}{c}{ August } \\
$59.4 \pm 2.6$ & $47.4 \pm 2.6$ & $45.6 \pm 3.6$ \\
$62.2 \pm 2.6$ & $55.2 \pm 2.6$ & $50.2 \pm 3.6$ \\
$64.8 \pm 2.6$ & $45.7 \pm 2.6$ & $45.3 \pm 2.6$ \\
$62.1_{\mathrm{a}} \pm 1.5$ & $49.4_{\mathrm{b}} \pm 1.5$ & $47.0_{\mathrm{b}} \pm 1.9$
\end{tabular}

\begin{tabular}{|c|c|c|c|c|c|}
\hline Autumn $^{3}$ & $\begin{array}{c}\text { Alkar Tall } \\
\text { wheatgrass }\end{array}$ & $\begin{array}{l}\text { Prairieland } \\
\text { Altai wildrye }\end{array}$ & $\begin{array}{c}\text { Bozoisky } \\
\text { Russian wildrye }\end{array}$ & $\begin{array}{c}\text { NewHy Hybrid } \\
\text { wheatgrass }\end{array}$ & $\begin{array}{c}\text { Native } \\
\text { rangeland }\end{array}$ \\
\hline 2000 & $45.4_{d}{ }^{B 4} \pm 1.5$ & $63.7_{\mathrm{a}}^{\mathrm{A}} \pm 2.1$ & $58.4_{b}^{\mathrm{B}} \pm 1.5$ & $52.4_{c}{ }^{\mathrm{A}} \pm 1.5$ & $45.8_{d}{ }^{B} \pm 2.1$ \\
\hline 2001 & $50.8_{b}{ }^{A} \pm 1.5$ & $63.0^{\mathrm{A}} \pm 2.1$ & $60.0_{\mathrm{a}}^{\mathrm{AB}} \pm 1.5$ & $51.7_{\mathrm{b}}^{\mathrm{A}} \pm 1.5$ & $44.3_{c}{ }^{B} \pm 2.1$ \\
\hline 2002 & $48.7_{c}^{\mathrm{AB}} \pm 1.5$ & $58.1_{a b}{ }^{A} \pm 2.5$ & $62.7_{\mathrm{a}}^{\mathrm{A}} \pm 1.5$ & $52.4_{b c}{ }^{A} \pm 1.5$ & $51.8_{c}{ }^{A} \pm 2.1$ \\
\hline
\end{tabular}

${ }^{1}$ Nonsignificant pasture-by-year interaction for spring pastures $(P=0.135)$. Significant pasture $(P=0.052)$ and year $(P=0.001)$ main effects. Pasture means with the same lowercase subscript are not different $(P=0.05)$. Year means with the same uppercase superscript are not different $(P=0.05)$.

${ }^{2}$ Nonsignificant date-by-year interaction $(P=0.313)$ for the summer pastures. Significant date main effect $(P=0.001)$. Date means with the same lowercase subscript are not different $(P=0.05)$

${ }^{3}$ Significant pasture-by-year interaction $(P=0.038)$ for the autumn pastures.

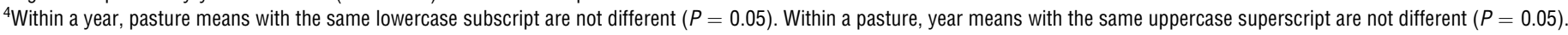


Table 6. Least-square means \pm standard error for average daily gains of yearling heifers on seeded pastures and native rangeland at Miles City, Montana.

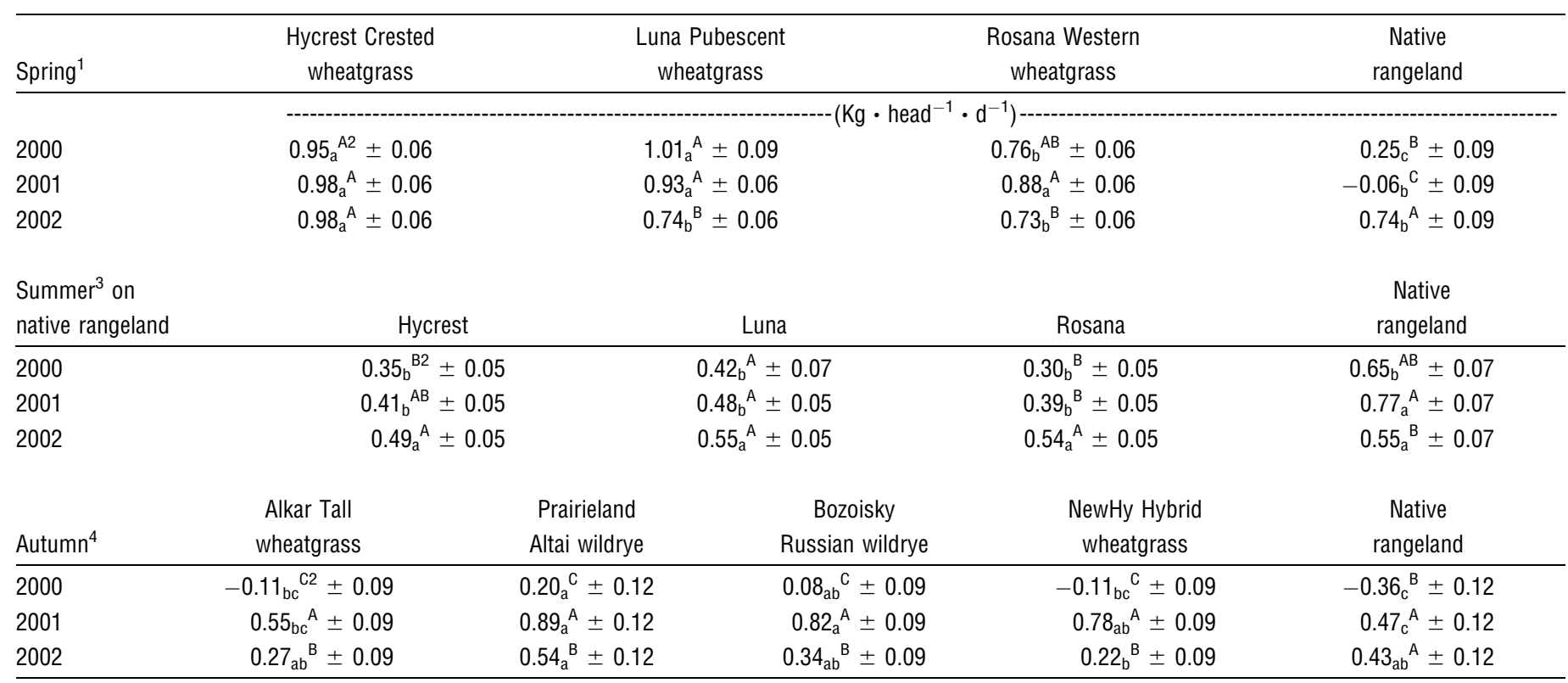

${ }^{1}$ Significant pasture-by-year interaction $(P=0.001)$ for spring gains.

${ }^{2}$ Within a pasture, year means with the same uppercase superscript are not different $(P=0.05)$. Within a year, pasture means with the same lowercase subscript are not different $(P=0.05)$. ${ }^{3}$ Significant pasture-by-year interaction $(P=0.021)$ per spring pastures and rangeland on summer gains.

${ }^{4}$ Significant pasture-by-year interaction $(P=0.008)$ for autumn gains.

rangeland (Table 3). No clear pattern evolved for CP concentration between the beginning and the end of the grazing period, remaining the same in $2000(P=0.755)$, declining in $2001(P=0.001)$ and increasing in $2002(P=0.001)$.
Summer. Crude protein concentration of standing crop declined $(P=0.001)$ as summer grazing progressed (Table 3$)$. $\mathrm{CP}$ was also greater in $2001(P=0.013)$ and 2002 when compared with 2000 (Table 3).

Table 7. Least-square means \pm standard error for average weight gains of yearling heifers while grazing on seeded pastures and native rangeland at Miles City, Montana.

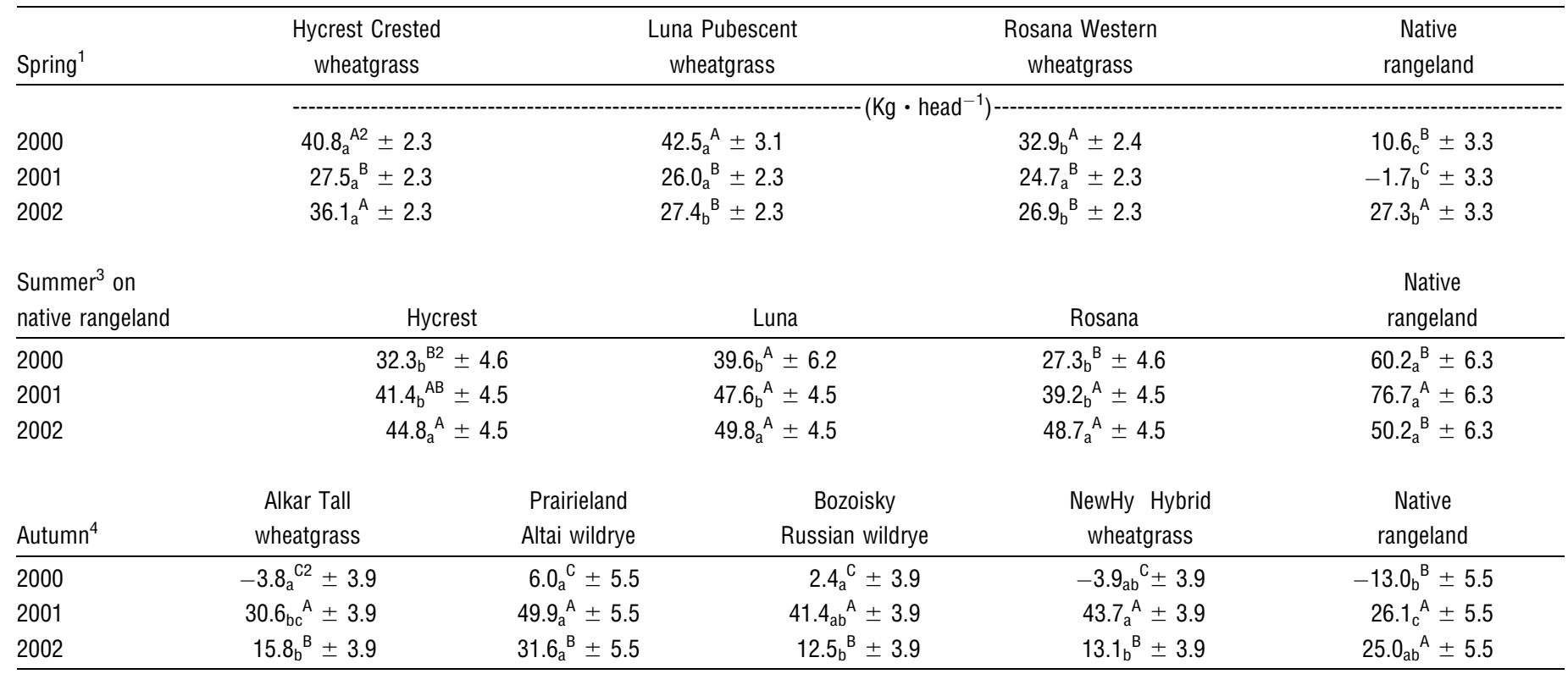

${ }^{1}$ Significant pasture-by-year interaction $(P=0.001)$ for spring gains.

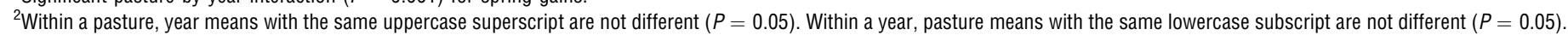
${ }^{3}$ Significant pasture-by-year interaction $(P=0.012)$ per spring pastures and rangeland on summer gains.

${ }^{4}$ Significant pasture-by-year interaction $(P=0.001)$ for autumn gains. 
Table 8. Least-square means \pm standard error for average gain per hectare of yearling heifers grazing on seeded pastures and native rangeland at Miles City, Montana.

\begin{tabular}{|c|c|c|c|c|c|}
\hline Spring $^{1}$ & $\begin{array}{c}\text { Hycrest Crested } \\
\text { wheatgrass }\end{array}$ & $\begin{array}{c}\text { Luna Pubescent } \\
\text { wheatgrass }\end{array}$ & $\begin{array}{c}\text { Rosana Western } \\
\text { wheatgrass }\end{array}$ & $\begin{array}{c}\text { Native } \\
\text { rangeland }\end{array}$ & Mean \\
\hline & 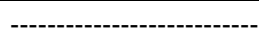 & 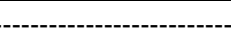 & $\left(\mathrm{Kg} \cdot \mathrm{ha}^{-1}\right)$ & 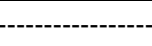 & |--------------. \\
\hline 2000 & $95.1 \pm 5.7$ & $98.9 \pm 7.9$ & $77.0 \pm 5.7$ & $0.8 \pm 8.1$ & $67.9^{A} \pm 3.5$ \\
\hline 2001 & $64.1 \pm 5.7$ & $60.6 \pm 5.7$ & $57.6 \pm 5.7$ & $-0.1 \pm 8.1$ & $45.5^{B} \pm 3.2$ \\
\hline 2002 & $84.2 \pm 5.7$ & $63.9 \pm 5.7$ & $62.9 \pm 5.7$ & $2.3 \pm 8.1$ & $53.3^{B} \pm 3.2$ \\
\hline Mean & $81.1_{\mathrm{a}} \pm 4.1$ & $74.5_{\mathrm{a}} \pm 4.4$ & $65.8_{\mathrm{a}} \pm 4.1$ & $1.0_{b} \pm 5.7$ & \\
\hline $\begin{array}{l}\text { Summer }{ }^{2} \text { on } \\
\text { native rangeland }\end{array}$ & Hycrest & Luna & Rosana & $\begin{array}{c}\text { Native } \\
\text { rangeland }\end{array}$ & Mean \\
\hline 2000 & $4.7 \pm 0.6$ & $5.8 \pm 0.9$ & $4.0 \pm 0.6$ & $8.8 \pm 0.9$ & $5.8^{\mathrm{B}} \pm 0.4$ \\
\hline 2001 & $6.6 \pm 0.6$ & $7.6 \pm 0.6$ & $6.3 \pm 0.6$ & $12.3 \pm 0.9$ & $8.2^{\mathrm{A}} \pm 0.4$ \\
\hline 2002 & $7.0 \pm 0.6$ & $7.8 \pm 0.6$ & $7.6 \pm 0.6$ & $7.8 \pm 0.9$ & $7.5^{\mathrm{A}} \pm 0.4$ \\
\hline Mean & $6.1_{b} \pm 0.5$ & $7.0_{\mathrm{ab}} \pm 0.5$ & $6.0_{b} \pm 0.5$ & $9.6_{a} \pm 0.7$ & \\
\hline
\end{tabular}

\begin{tabular}{|c|c|c|c|c|c|}
\hline Autumn ${ }^{3}$ & $\begin{array}{c}\text { Alkar Tall } \\
\text { wheatgrass }\end{array}$ & $\begin{array}{l}\text { Prairieland } \\
\text { Altai wildrye }\end{array}$ & $\begin{array}{c}\text { Bozoisky } \\
\text { Russian wildrye }\end{array}$ & $\begin{array}{c}\text { NewHy Hybrid } \\
\text { wheatgrass }\end{array}$ & $\begin{array}{c}\text { Native } \\
\text { rangeland }\end{array}$ \\
\hline 2000 & $-8.1_{\mathrm{a}}{ }^{\mathrm{C} 4} \pm 7.5$ & $12.9^{C} \pm 10.6$ & $5.2_{\mathrm{a}}^{\mathrm{C}} \pm 7.5$ & $-8.5_{a}^{C} \pm 7.5$ & $-11.1_{\mathrm{a}}^{\mathrm{A}} \pm 10.6$ \\
\hline 2001 & $66.2_{\mathrm{b}}^{\mathrm{A}} \pm 7.5$ & $107.9^{A} \pm 10.6$ & $89.5_{\mathrm{ab}}{ }^{\mathrm{A}} \pm 7.5$ & $94.4^{\mathrm{A}} \pm 7.5$ & $5.1_{c}^{A} \pm 10.6$ \\
\hline 2002 & $34.1_{\mathrm{b}}^{\mathrm{B}} \pm 7.5$ & $68.2^{\mathrm{B}} \pm 10.6$ & $27.0_{b c}{ }^{B} \pm 7.5$ & $28.2_{b c}{ }^{B} \pm 7.5$ & $1.8_{C}{ }^{A} \pm 10.6$ \\
\hline
\end{tabular}

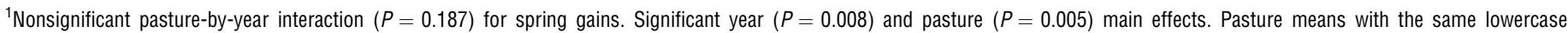
subscript are not different $(P=0.05)$. Year means with the same uppercase superscript are not different $(P=0.05)$.

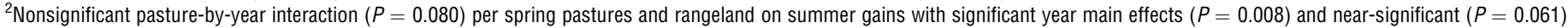
pasture main effects. Pasture means with the same lowercase subscript are not different $(P=0.05)$. Year means with the same uppercase superscript are not different $(P=0.05)$. ${ }^{3}$ Significant pasture-by-year interaction $(P=0.011)$ for autumn gains.

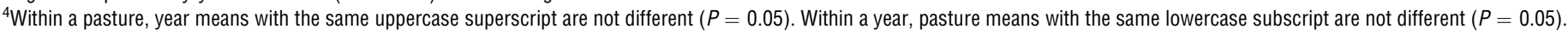

Autumn. Crude protein was highest in Bozoisky and Prairieland (2000 and 2001), intermediate in NewHy and native rangeland, and lowest in Alkar $(P=0.001)$ (Table 3). The significant pasture-by-date interaction $(P=0.001)$ revealed that $\mathrm{CP}$ was similar between the beginning and end of the grazing period for Alkar $(P=0.086)$ and native rangeland $(P=0.711)$ but declined significantly for Prairieland $(P=0.001)$, Bozoisky $(P=0.001)$, and NewHy $(P=0.001)$.

\section{Diet Quality}

Spring. Crude protein content of diets, as represented by esophageal extrusa, was consistently greater for Hycrest than native rangeland $(P=0.076)$ (Table 4). Ranking of diet $\mathrm{CP}$ for Rosana with other pastures varied among years $(P=0.058)$. Diet quality did not vary among years for the other seeded pastures or native rangeland $(P=0.186)$. Digestibility of diets was similar among seeded pastures $(P=0.056)$ (Table 5$)$ averaging more than $70 \%$; it was only slightly less on native rangeland.

Summer. Dietary CP concentrations declined from June to July $(P=0.001)$, remained stable through August (Table 4), and were greatest $(P=0.001)$ in 2001 , intermediate in 2002, and lowest in 2000. Diet digestibilities declined $(P=0.001)$ from June to July and August (Table 5) on native rangeland.

Autumn. Diets contained greater CP on Prairieland pastures in $2000(P=0.002)$ and Bozoisky pastures in 2002 $(P=0.007)$ (Table 4$)$. CP varied among years but was greater in 2001 and 2002 than 2000 on Bozoisky $(P=0.026)$, NewHy
$(P=0.008)$, and native rangeland $(P=0.026)$ pastures. Autumn diets consistently were greater $(P=0.009)$ in IVDOM on Prairieland and Bozoisky compared with the other pastures. IVDOM of diets was greater $(P=0.046)$ on NewHy pastures than the similar $(P=0.540)$ IVDOM of diets on native rangeland and Alkar pastures.

\section{Livestock Performance}

Spring. Daily gains $(P=0.001)$ and gains per head $(P=0.001)$ were greater on all seeded pastures compared with native rangeland in 2000 and 2001 (Tables 6 and 7). In 2002 , heifer gains were greater $(P=0.033)$ on Hycrest than other seeded pastures or native rangeland. Gains were similar $(P=0.890)$ among the other pastures. Gains per hectare were similar $(P=0.076)$ among seeded pastures, ranging from 66 to $81 \mathrm{~kg} \cdot \mathrm{ha}^{-1}$, and were greater in 2000 than in either 2001 $(P=0.003)$ or $2002(P=0.016)$ (Table 8$)$.

Summer. Gain per day and, equivalently, gains per head $(P=0.021)$ were greater for heifers that previously grazed native rangeland compared with those having grazed seeded pastures in spring 2000 and 2001, but gains were similar $(P=0.410)$ among spring pastures in 2002 (Tables 6 and 7). No clear pattern was observed for gains among years for the various pastures. Gains per hectare during summer were greatest for heifers previously grazing native rangeland $(P=0.053)$ with intermediate gains on Luna (Table 8$)$. Greater gains per hectare occurred in $2001(P=0.003)$ and 2002 
$(P=0.012)$ than 2000 (Table 8$)$. When spring + summer gains were compared, the effect of spring pastures was not significant $(P=0.244)$. Spring + summer gains averaged $0.55 \pm 0.01$ $\mathrm{kg} \cdot \mathrm{head}^{-1} \cdot \mathrm{d}^{-1}$ and $72.6 \pm 1.4 \mathrm{~kg} \cdot \mathrm{head}^{-1}$.

Autumn. Greatest daily gains (Table 6) occurred on Prairieland $(P=0.039)$ pastures in 2000 . Daily gains on Bozoisky pastures were greater $(P=0.004)$ than on Alkar or native rangeland (Table 6) but similar $(P=0.118)$ to those on other seeded pastures. In 2001, daily gains were greater on Prairieland $(P=0.022)$, Bozoisky $(P=0.022)$, and NewHy $(P=0.057)$ than on Alkar or native rangeland. Gains were greater $(P=0.034)$ on Prairieland than NewHy in 2002 . Daily gains were greatest $(P=0.001)$ in 2001, intermediate in 2002, and least in 2000. Total gains per head (Table 7) responded similarly to daily gains in 2001 (Table 6). However, in 2000, total gains on native rangeland were not different $(P=0.185)$ from those on NewHy, and in 2002, total gains on native rangeland were similar $(P=0.067)$ to those on seeded pastures. Gains per hectare were similar $(P=0.155)$ among pastures in 2000, whereas gains were greater on Prairieland $(P=0.018)$ and NewHy $(P=0.037)$ than Alkar in 2001 and greater for Prairieland $(P=0.039)$ than all other pastures in 2002 (Table 8).

\section{DISCUSSION}

Seedings of cool-season cultivars are proposed as a tool to increase available forage and to extend the period of high quality forage for grazing livestock (Vallentine 1980; Wasser 1982; Holzworth et al. 2003). Data from this study and others clearly show that seeded pastures can provide more forage than native rangeland (Smoliak 1968; Hart et al. 1983; Adams et al. 1989) and will support increased grazing pressure in spring and autumn. However, this may not occur every year or with every seeded species (Willms and Jefferson 1993). The utility of seeded forages depends in part on the vigor of the seeded stand, the adaptability of the seeded forage to a given site (e.g., Luna and Bozoisky in this study), and on the amount and distribution of precipitation.

Livestock performance varies among seeded pastures and native rangeland (Houston and Urick 1972; Hart et al. 1983; Adams et al. 1989; Hofmann et al. 1993). Some report increased individual animal performance from grazing seeded pastures (Houston and Urick 1972) whereas others report the main advantage to be associated with increased seasonal carrying capacity (Hart et al. 1983; Adams et al. 1989; Hofmann et al. 1993). We observed both increased animal performance and potential increases in spring and autumn carrying capacity with seeded pastures during most years of this study.

Spring gains of heifers were similar to previously reported gains of heifers $\left(0.52\right.$ to $0.78 \pm 0.04 \mathrm{~kg} \cdot \mathrm{head}^{-1} \cdot \mathrm{day}^{-1}$ and $72 \pm 7.2 \mathrm{~kg} \cdot \mathrm{ha}^{-1}$ ) grazing Hycrest, Luna, and Rosana at this location (Haferkamp et al. 2002). Spring gains on native rangeland were generally lower than on seeded pasture.

Summer gains of heifers that grazed seeded pastures in the spring were somewhat less than those continuously grazing native rangeland. Summer gains of heifers from the spring native rangeland treatment were similar to previously reported gains of 0.3 to $0.8 \mathrm{~kg} \cdot \mathrm{d}^{-1}$ but somewhat lower than 3 to 29 $\mathrm{kg} \cdot \mathrm{ha}^{-1}$ for yearling steers throughout a summer grazing period (Heitschmidt et al. 1993). As forage quality declines on native rangeland throughout the summer, heifers that gained more rapidly in the spring may have a more difficult time obtaining an adequate amount of nutrients to maintain weight gain equal to that of the heifers that grew more slowly in the spring. In some conditions, late summer grazing has resulted in weight loss in yearling cattle on Northern Great Plains rangelands (Currie et al. 1989), but this has not occurred in years with more favorable growing conditions (Grings et al. 2002).

Improved gains on Prairieland and Bozoisky compared with native rangeland in the autumn may be indicative of a viable management strategy for pregnant heifers going into winter. Strategies that increase weight and condition of pregnant cattle before winter may reduce winter feed requirements. The relative economic value of these seeded pastures compared with native rangeland and the impact on harvested feed needs were not evaluated in this study.

Spring gains followed a generalized pattern of forage quality among the seeded forages. Hycrest and Luna generally showed greater CP and IVDOM in diet samples with correspondingly greater weight gain in heifers. Increased gains for heifers grazing Prairieland and Bozoisky in the autumn were also reflective of the increased dietary CP and IVDOM for these forages.

In addition to improved forage quality in seeded pastures, some differences in heifer gain could be related to increased energy expenditures for grazing heifers on native rangeland. Seeded pastures were flat and relatively small, whereas the native rangeland pasture was larger with undulating terrain. Estimates for energy expenditures for grazing in extensive environments have been shown to increase energy needs by as much as $25 \%-35 \%$ (Osuji 1974) compared with stallfed animals.

Annual variation in amount and distribution of precipitation appears to explain some, but not all, of the variation in standing crop, forage and diet nutritive quality, and livestock performance. Above average precipitation in October and November 2000 and June and July 2001 potentially increased standing crops during summer and on some pastures during autumn 2001. The above average precipitation in June and July 2001 also appeared to increase CP concentrations in forage as well as CP and IVDOM in diets through the summer of 2001 and at the turn-in date for some of the autumn pastures. The above average precipitation in May, August, and September 2002 did not appear to stimulate increases in standing crop or forage and diet nutritive quality except in a few cases.

Selecting suitable plant species for seasonal grazing is an important aspect of developing a viable integrated forage system. Earlier, Haferkamp et al. (2002) discussed the problems with maintaining stands of pubescent wheatgrass in the 335-mm precipitation zone in the Northern Great Plains. The 4 forages that were grazed in autumn reportedly have very good to excellent salinity tolerance, are adapted to heavy textured soils, and are recommended for autumn grazing (Holzworth et al. 2003). However, findings from this study suggest that Bozoisky Russian wildrye is not as well adapted to the high $\mathrm{pH}$ of Marias clay and silty clay soils as the other cultivars evaluated. Also, use of Alkar tall wheatgrass during autumn does not take advantage of the pattern of forage pro- 
duction and nutritive quality of this grass. Much of the standing crop available to heifers in autumn was the stiff reproductive shoots produced during spring and early summer by this species. The low forage and diet quality for this species emphasize this conclusion.

\section{MANAGEMENT IMPLICATIONS}

Seeded pastures of cool-season grasses provide livestock managers on western rangelands an opportunity to potentially increase available forage and provide quality forage for grazing livestock. Choosing the best forage for the intended use is critical. In this study, Luna, Alkar, and Bozoisky were probably not the best-adapted cultivars for the proposed use on these soils at this location.

Findings of this study and others clearly show that seasonal livestock gains may be better on seeded pastures than on native rangeland even with increased stocking pressure. Thus, seeded pastures can be an effective management tool that defers use of native rangeland. Results of this study suggest Hycrest crested wheatgrass would be the best among the cultivars evaluated for grazing in spring, and Prairieland Altai wildrye would, likewise, be the most productive pasture for autumn. These findings, based on livestock performance, validate recommendations regarding use of complimentary forages based on their agronomic characteristics. However, early spring gains may not be maintained when cattle are moved from seeded pastures to native rangeland for the summer grazing season, and the increased gains may not occur every year. Livestock managers may need to modify their tactics to take full advantage of increased gains on seeded pastures.

\section{ACKNOWLEDGMENTS}

The authors express appreciation to the USDA-ARS Forage and Range Research Laboratory, Logan, Utah, for providing seed for this study and personnel of USDA-ARS and Montana Agr. Exp. Sta. located at Fort Keogh Livestock and Range Research Laboratory for their diligent efforts in this research.

\section{LITERATURE CITED}

Adams, D. C., R. B. StaigmilleR, and B. W. Knapp. 1989. Beef production from native and seeded Northern Great Plains ranges. Journal of Range Management 42:243-247.

Currie, P. O., J. D. Volesky, D. C. Adams, and B. W. Knapp. 1989. Growth patterns of yearling steers determined from daily live weights. Journal of Range Management 46:529-533

Grings, E. E., R. K. Heitschmidt, R. E. Short, and M. R. Haferkamp. 2002. Intensiveearly stocking for yearling cattle in the Northern Great Plains. Journal of Range Management 55:135-138.

Haferkamp, M. R., E. E. Grings, R. K. Heitschmidt, and M. D. MacNeil. 2002. Quality and persistence of forages in the Northern Great Plains. Journal of Range Management 55:482-487.
Hart, R. H., J. W. Waggoner, D. H. Clark, C. C. Kaltenbach, J. A. Hager, and M. B. MARSHALL. 1983. Beef cattle performance on crested wheatgrass plus native range vs. native range alone. Journal of Range Management 36:38-40.

Heitschmidt, R. K., J. D. Volesky, M. R. Haferkamp, and P. O. CurRie. 1993. Steer performance on native and modified Northern Great Plains rangeland. Journal of Range Management 46:529-533.

Hofmann, L., R. E. Ries, J. F. Karn, and A. B. Frank. 1993. Comparison of seeded and native pastures grazed from mid-May through September. Journal of Range Management 46:251-254.

Holzworth, L., J. Mosley, D. Cash, D. Koch, and K. Crane. 2003. Dryland pastures in Montana and Wyoming. Montana State University Ext. Serv. EB 19. 32 p. Available at: http://www.montana.edu/wwwpb/pubs/eb19.pdf. Accessed 28 December 2004

Houston, W. R., AND J. J. URICK. 1972. Improved spring pastures, cow-calf production, and stocking rate carryover in the Northern Great Plains. Washington, DC: USDA-ARS Tech. Bull. No. 1451. 21 p.

Karn, J. F., R. E. Reis, and L. Hofmann. 1999. Season-long grazing of seeded coolseason pastures in the Northern Great Plains. Journal of Range Management $52: 235-240$

Kilcher, M. R., and T. Lawrence. 1979. Spring and summer pastures for southwestern Saskatchewan. Canadian Journal of Plant Science 59:339-342.

KüCHLER, A. W. 1964. Potential natural vegetation of the conterminous United States. American Geographical Society Spec. Pub. 36. New York, NY: American Geographical Society. 77 p.

Mortenson, M. C. 2003. Effects of interseeded alfalfa (Medicago sativa spp. falcata) on forage production, forage quality, and carbon sequestration on a mixed-grass prairie rangeland [thesis], Laramie, WY: University of Wyoming. $59 \mathrm{p}$.

[NOAA] National Oceanic and Atmospheric Administration. 2000-2002. Climatological data annual summary, Montana. 2000-2002:13. Asheville, NC: National Climatological Data Center.

OsuJI, P. 0. 1974. The physiology of eating and the energy expenditure of the ruminant at pasture. Journal of Range Management 27:437-443.

SAS Online Doc [Computer Program]. 2002. Version 8. Cary, NC: SAS Institute, Inc. SmoLIAK, S. 1968. Grazing studies on native range, crested wheatgrass, and Russian wildrye pastures. Journal of Range Management 21:47-50.

Smoliak, S., AND S. B. SLeN. 1974. Beef production on native range, crested wheatgrass, and Russian wildrye pastures. Journal of Range Management 27:433-436.

Soll Conservation Service. 1983. Technical guide, Section II-E-8. 10-14" precipitation zone. Eastern Sedimentary Plains, Montana.

Tilley, J. M. A., and R. A. TerRy. 1963. A two-stage technique for the in vitro digestion of forage crops. Journal of British Grassland Society 18:104-111.

Vallentine, J. F. 1980. Range development and improvements. 2nd ed. Provo, UT: Brigham Young University Press. 545 p.

WASSER, C. H. 1982. Ecology and culture of selected species useful in revegetating disturbed land in the west. Springfield, VA: Biological Services Program, Fish and Wildlife Serv., US Dept. of Interior. National Technical Information Service, US Dept. of Commerce. $349 p$.

White, L. M., G. P. Hartman, and J. W. Bergman. 1981. In vitro digestibility, crude protein, and phosphorus content of straw of winter wheat, spring wheat, barley, and oat cultivars in eastern Montana. Agronomy Journal 73: 117-121.

Wight, J. R., C. K. Gee, and R. J. Kartchner. 1983. Integrated rangeland and cropland management. Dryland agriculture. Madison, WI: ASA-CSSA-SSSA. Agronomy Monograph 23. p 435-460.

Willms, W. D., And P. G. Jefferson. 1993. Production characteristics of the mixed prairie: constraints and potential. Canadian Journal of Animal Science 73: 765-778. 